alveolar macrophages [2], as well as from the pulmonary fibroblasts [3]. Its action is basically opposed to that of TNF- $\alpha$, which is a rather inflammatory cytokine [2]. It is considered, as well as IL-4, a major IL in the T-helper cell type 2 immune response [4]. Interestingly, IL-10 levels have been found to be high in rat tissues with pulmonary fibrosis, while in rats with IL-10 deficiency, the inflammatory response has been found to be more intense than in the wild animals [5]. In in vitro studies, the addition of IL-10 in fibroblasts has lessened the expression of type 1 collagen, the production of which is stimulated by transforming growth factor- $\beta$ [6]. Those observations support the findings of BERGERON et al. [1], as well those from our own studies, that IL-10 might be an important target for future therapies, as well as a diagnostic tool.

We think that investigating the interleukin-10 pathways and possible therapeutic uses might be important in discovering more about idiopathic pulmonary fibrosis and providing patients with a more effective treatment. Moreover, serum and tissue cytokine profiles might be important in this direction.

\section{P.G. Tsoutsou*, K.I. Gourgoulianis ${ }^{\#}$}

*Nikis 33, Syntagma, Athens and ${ }^{\#}$ Medical school, University of Thessaly, Larissa, Greece.

\section{References}

1. Bergeron A, Soler P, Kambouchner $\mathrm{M}$, et al. Cytokine profiles in idiopathic pulmonary fibrosis suggest an important role for TGF- $\beta$ and IL-10. Eur Respir J 2003; 22: 69-76.

2. Martinez JA, King TE, Brown K, et al. Increased expression of the interleukin-10 gene by alveolar macrophages in interstitial lung disease. Am J Physiol 1997; 273: 676-683.

3. Vancheri C, Mastruzzo C, Tomaselli V, et al. Normal human lung fibroblasts differently modulate interleukin-10 and interleukin-12 production by monocytes: implications for an altered immune response in pulmonary chronic inflammation. Am J Respir Cell Mol Biol 2001; 25: 592-599.

4. Zhou Y, Giscombe R, Huang D, Levfert AK. Novel genetic association of Wegener's granulomatosis with the interleukin 10 gene. J Rheumatol 2002; 29: 317-320.

5. Huaux F, Louahed J, Hudspith B, et al. Role of interleukin10 in the lung response to silica in mice. Am J Resp Cell Mol Biol 1998; 18: 51-59.

6. Arai $\mathrm{T}$, Abe $\mathrm{K}$, Matsuoka $\mathrm{H}$, et al. Introduction of the interleukin- gene into mice inhibited bleomycin- lung injury in vivo. Am J Physiol Lung Cell Mol Physiol 2000; 278: 914-922.

\section{From the authors.}

We thank P.G. Tsoutsou and K.I. Gourgoulianis for their interest in our manuscript in which we suggested a potential important role for transforming growth factor- $\beta$ and interleukin (IL)-10 in the pathogenesis of idiopathic pulmonary fibrosis (IPF) [1]. We also read with great interest the results they report concerning increased levels of IL-10 in the sera of their patients with IPF.
As they emphasised in their comments, the production of cytokines varies during the course of an inflammatory pulmonary process and it is very important to be precise about the moment of the study, which clearly corresponded in our work to "a given moment" of the development of IPF. All the tissue specimens studied were open lung biopsies obtained from patients with IPF at the time of histological confirmation of the diagnosis. It should be emphasised that no patient had a history or symptoms suggesting pulmonary fibrosis of a known cause and none had received any treatment for pulmonary fibrosis prior to lung biopsy.

In our study, we found that hyperplastic type II pneumocytes were the main source of IL-10 in the lung tissue specimens from patients with IPF [1]. One can speculate that at least a part of IL-10 produced in the lung in the course of IPF could circulate in the bloodstream, and thus could participate in the elevated levels of this cytokine in the sera of patients, as reported by P.G. Tsoutsou and K.I. Gourgoulianis. Additional work is needed, however, to determine the cellular sources of the increased circulating levels of IL-10 in IPF and to confirm that in the same patients, the serum and pulmonary levels of IL-10 are correlated.

The absence of increased levels of IL-10 in the blood of patients with known causes of pulmonary fibrosis raises concern about the histopathological pattern of the fibrotic process. Besides usual interstitial pneumonia, i.e. the histological counterpart of IPF, several other patterns have been identified in the spectrum of diffuse fibrotic interstitial pneumonias (such as desquamative interstitial pneumonia, nonspecific interstitial pneumonia, etc.) that can be associated with various aetiologies [2]. Cytokines involved in the pathogenesis of these patterns of pulmonary fibrosis might be different from cytokines present in IPF. Studies performed in situ on lung tissue samples from patients with secondary pulmonary fibrosis with precise histopathological pattern are needed to answer this question. In particular, the presence or absence and the extent of type II alveolar hyperplasia in these different interstitial pneumonias could also influence the amounts of IL-10 produced in the lung of these patients.

Finally, we agree with P.G. Tsoutsou and K.I. Gourgoulianis that studies evaluating the role of interleukin-10 in idiopathic pulmonary fibrosis may give further insights in the management of patients with this very disabling disorder.

\section{A. Tazi}

Service de pneumologie, Hôpital Avicenne, 125 rue de stalingrad, 93009 Bobigny, France.

\section{References}

1. Bergeron A, Soler $\mathrm{P}$, Kambouchner $\mathrm{M}$, et al. Cytokine profiles in idiopathic pulmonary fibrosis suggest an important role for TGF- $\beta$ and IL-10. Eur Respir J 2003; 22: 69-76.

2. ATS/ERS International multidisciplinary consensus classification of the idiopathic interstitial pneumonias. Am J Respir Crit Care Med 2002; 165: 277-304.

\title{
Spirometry in young children
}

\section{To the Editor:}

With interest we read the article by GRACCHI et al. [1]. In recent years we also evaluated the use of computer-animation programs for spirometry with children in our pulmonary function lab. However, we did not find a significant difference in maximal performance with or without the use of the "candles" and the "balloon" program. 
In addition we are surprised to see that the authors did find a difference in reproducibility. When the use of an animation program is only for additional support you are not likely to find a difference in reproducibility. It should be noted that an animation program will of course never be able to replace the lung function technician, but should be used as an extra incentive.

The exact circumstances of this study are not quite clear. Was the use of an incentive always accompanied by the same coaching as blowing without the use of an incentive?

In our study we did find an important difference: all children did find the lung function test more attractive with the use of a computer-animation, and as a result we were able to have pulmonary function tests done in younger children.

So we came to the conclusion that the use of a computeranimation program is useful, even for routine use.

\section{Steenbruggen, R.J. Roorda}

Dept of Paedriatrics, Isala klinieken, Zwolle, the Netherlands.

\section{References}

1. Gracchi V, Boel M, van der Laag J, van der Ent CK. Spirometry in young children: should computer-animation programs be used during testing? Eur Respir J 2003; 21: 872-875.

From the authors:

We thank I. Steenbruggen and R.J. Roorda for their attention and comments on our article on the use of computer animation programs during maximal expiratory flow volume (MEFV) measurements in young children [1]. We were also surprised by our finding that the use of the programs did not improve maximal effort. As paediatricians we always think that "toys are good for children". However, our data show that MEFV measurements require serious effort and that "toys are no good for the results".

In our study the use of the incentive was always accompanied by the same coaching by the same lung function technician. Our findings of less performance on forced vital capacity with use of the incentives were significant and very consistent for both programs (balloons and candles), for both younger and older children and for both children with and without experience. Because I. Steenbruggen and R.J. Roorda do not present any data on their findings we cannot rule out whether their lack of significant findings was merely a power problem or not.

MEFV measurements are only reproducible when the performance is really maximal, i.e. when flow limitation is achieved. Sub-optimal MEFV performance will therefore always be accompanied by a loss of reproducibility, as was observed in our patients.

We agree that children find the lung function test more attractive with use of the programs. This was also observed by NySTAD et al. [2]. So our message is: "first play the game and then do the job" (or the other way round), but do not try to do them together.

V. Gracchi, M. Boel, J. van der Laag, C.K. van der Ent Dept of Pediatric Respiratory Diseases, University Medical Center, Utrecht, the Netherlands.

\section{References}

1. Gracchi V, Boel M, Van der Laag J, Van der Ent CK. Spirometry in young children: should computer-animation programs be used during testing? Eur Respir J 2003; 21: 872875.

2. Nystad W, Samuelsen SO, Nafstad P, Edvardsen E, Stensrud T, Jaakkola JJ. Feasibility of measuring lung function in preschool children. Thorax 2002; 57: 1021-1027. 